\section{(2) OPEN ACCESS}

\title{
Enhanced oxidative stress and presence of ventricular aneurysm for risk prediction in cardiac sarcoidosis
}

\author{
Ryosuke Yoshitomi, ${ }^{1}$ Shigeki Kobayashi (i) , ${ }^{1}$ Yasutake Yano, ${ }^{1}$ Yusuke Nakashima, \\ Shohei Fujii, ${ }^{1}$ Takuma Nanno, ${ }^{1}$ Hironori Ishiguchi, ${ }^{1}$ Masakazu Fukuda, ${ }^{1}$ \\ Yasuhiro Yoshiga, ${ }^{1}$ Takayuki Okamura, ${ }^{1}$ Kazuyoshi Suga, ${ }^{2}$ Reo Kawano, ${ }^{3}$ \\ Masafumi Yano ${ }^{1}$
}

\begin{abstract}
- Additional supplemental material is published online only. To view, please visit the journal online (http://dx.doi. org/10.1136/heartjnl-2021320244)
\end{abstract}

${ }^{1}$ Department of Medicine and Clinical Science, Yamaguchi University Graduate School of Medicine, Ube, Japan

${ }^{2}$ Department of Radiology, St Hill Hospital, Ube, Japan

${ }^{3}$ Clinical Research Center in Hiroshima, Hiroshima University Hospital, Hiroshima, Japan

\section{Correspondence to}

Dr Shigeki Kobayashi,

Department of Medicine and Clinical Science, Yamaguchi University Graduate School of Medicine, Ube 755-8505,

Yamaguchi, Japan;

skoba@yamaguchi-u.ac.jp

RY and SK contributed equally.

Received 29 August 2021 Accepted 13 December 2021 Published Online First 25 January 2022

\section{Linked}

- http://dx.doi.org/10.1136/ heartjnl-2021-320590

Check for updates

(c) Author(s) (or their employer(s)) 2022. Re-use permitted under CC BY-NC. No commercial re-use. See rights and permissions. Published by BMJ.

To cite: Yoshitomi $\mathrm{R}$ Kobayashi S, Yano Y, et al. Heart 2022;108:429-437.

\section{ABSTRACT}

Objective Sudden cardiac death $(S C D)$ is the major cause of death in cardiac sarcoidosis (CS). We aimed to identify the prognostic markers for sustained ventricular tachycardia (SVT) and SCD in patients with CS.

Methods We performed a prospective observational cohort study for patients with CS diagnosed according to the Japanese or Heart Rhythm Society guidelines between June 2008 and March 2020 in our hospital. The primary endpoint was a composite of the first SVT and SCD. The levels of urinary 8-hydroxy-2'-deoxyguanosine (U-8-OHdG), a marker of oxidative DNA damage that reflects the inflammatory activity of CS, other biomarkers, and indices of cardiac function and renal function were measured on admission.

Results Eighty-nine consecutive patients with CS were enrolled; 28 patients with no abnormal ${ }^{18} \mathrm{~F}$ fluorodeoxyglucose $\left({ }^{18} \mathrm{~F}-\mathrm{FDG}\right)$ accumulation in the heart were excluded and 61 patients with abnormal ${ }^{18} \mathrm{~F}$-FDG accumulation were followed up for a median of 46 months (IQR: 20-84). During the follow-up period, 15 of 61 patients showed SVT $(n=12)$ or SCD $(n=3)$. A Cox proportional hazard model showed that U-8-OHdG concentration and presence of ventricular aneurysm (VA) were independent predictors of first SVT/SCD. The cut-off U-8-OHdG concentration for predicting first SVT/SCD was $14.9 \mathrm{ng} / \mathrm{mg} \cdot \mathrm{Cr}$. Patients with U-8-OHdG concentration $\geq 14.9 \mathrm{ng} / \mathrm{mg} \cdot \mathrm{Cr}$ and VA showed a significantly increased risk of SVT/SCD.

Conclusions U-8-OHdG and presence of VA were powerful predictors of first SVT/SCD in patients with CS, facilitating the stratification of cardiac events and providing relevant information about the substrates of ventricular tachycardia.

\section{INTRODUCTION}

Sarcoidosis is a systemic inflammatory disease characterised by non-caseating epithelioid granulomas of unknown aetiology. Cardiac sarcoidosis (CS) presents with a poor prognosis and is often associated with heart failure (HF), high-degree atrioventricular block and ventricular arrhythmia. ${ }^{1}$ Recent studies have shown that patients with CS have an increased risk of sudden cardiac death (SCD), which accounts for most lethal ventricular arrhythmias. ${ }^{1-3}$ With new modalities, relevant prognostic information from techniques such as ${ }^{18} \mathrm{~F}$-fluorodeoxyglucose $\left({ }^{18} \mathrm{~F}\right.$-FDG)-positron emission tomography (PET), cardiac magnetic resonance imaging and programmed electrical stimulation has been reported as useful in predicting fatal arrhythmias in CS. ${ }^{4-8}$

8-hydroxy-2'-deoxyguanosine (8-OHdG), an oxidative stress marker, is produced by oxidation of 2'-deoxyguanosine, a DNA base, under cellular oxidative stress conditions. ${ }^{9}$ During DNA repair, 8 -OHdG is excreted into the blood and passed into the urine without metabolism. Therefore, the 8-OHdG concentrations in blood and urine can be precisely measured by ELISA using an anti-8-OHdG antibody (N45.1; figure $1 \mathrm{~A}) .^{9-12}$ Blood sampling data obtained from the coronary sinus and aorta showed that the 8-OHdG concentration was significantly higher in the coronary sinus than the aorta in heart disease complicated with HF, suggesting that $8-\mathrm{OHdG}$ was produced in the cardiac tissue. ${ }^{9}$ Interestingly, the difference in $8-\mathrm{OHdG}$ concentrations between the coronary sinus and aorta was much larger in patients with active CS than those with non-active CS and dilated cardiomyopathy, ${ }^{10}$ suggesting that CS-induced inflammation strongly enhances cardiac oxidative stress.

Furthermore, urinary 8-OHdG (U-8-OHdG) levels are significantly correlated with the inflammatory activity observed on ${ }^{18} \mathrm{~F}$-FDG-PET in $\mathrm{CS}^{9-11}$ as well as the degree of oxidative stress in myocardial biopsy tissue. ${ }^{12}$ We previously reported that U-8OHdG levels had a significant positive correlation with myocardial fibrosis. ${ }^{12}$ In addition, U-8-OHdG levels were significantly higher in CS patients with ventricular tachycardia (VT) than in those without, indicating a VT-related increase in U-8-OHdG concentration. ${ }^{12}$ So far, no biomarkers have been reported to predict fatal arrhythmias and SCD in patients with CS. ${ }^{13}$ In this prospective cohort study, we investigated the markers evaluated at the time of CS diagnosis that could serve as predictors of sustained ventricular tachycardia (sVT)/SCD.

\section{METHODS}

\section{Study design and patient population}

This was a single-centre, observational, prospective study including consecutive patients admitted to our hospital for suspected CS and finally diagnosed with CS during hospitalisation. Briefly, a definitive diagnosis of CS was made based on the Japanese Circulation Society's or Heart Rhythm 

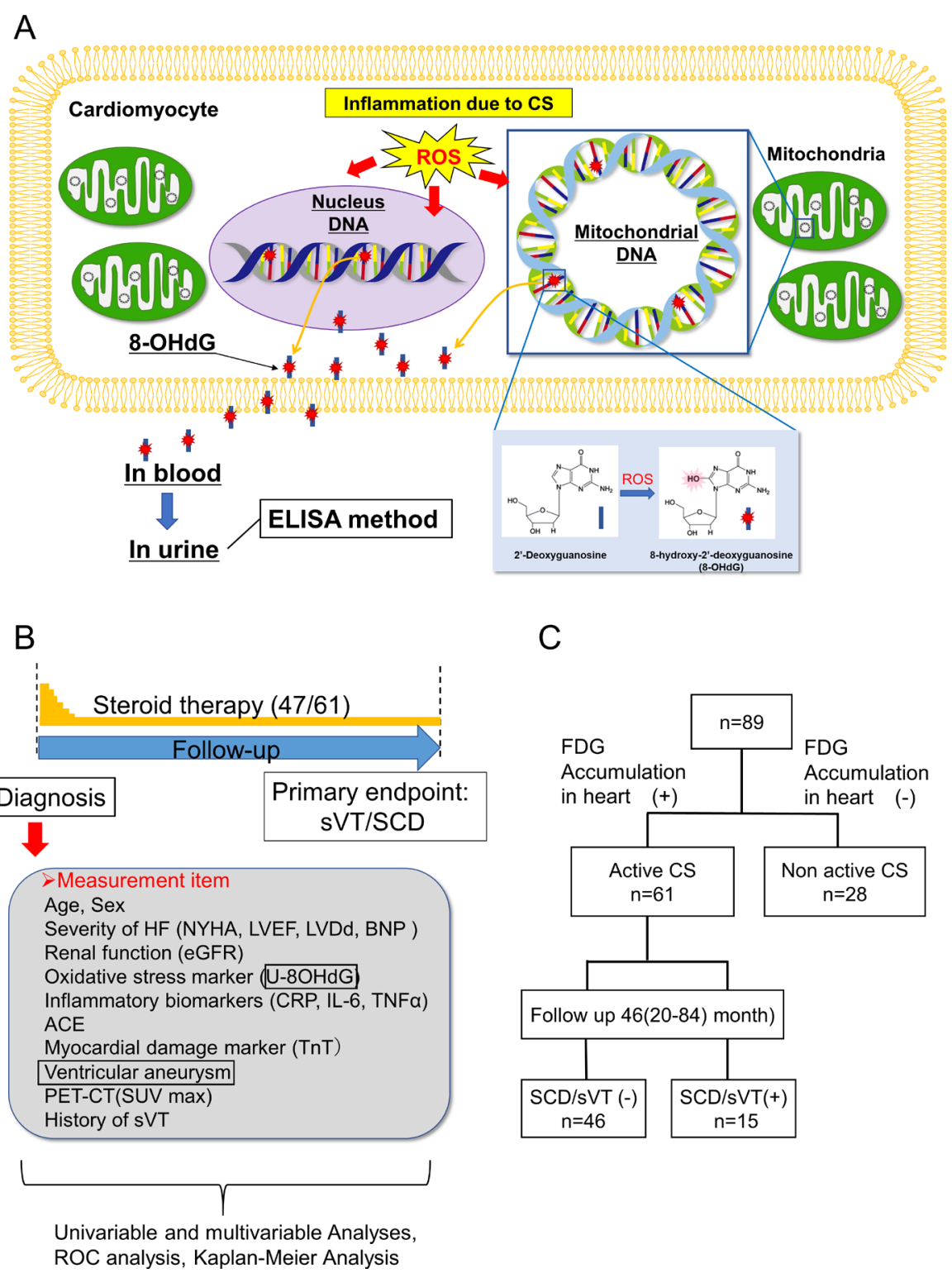

C

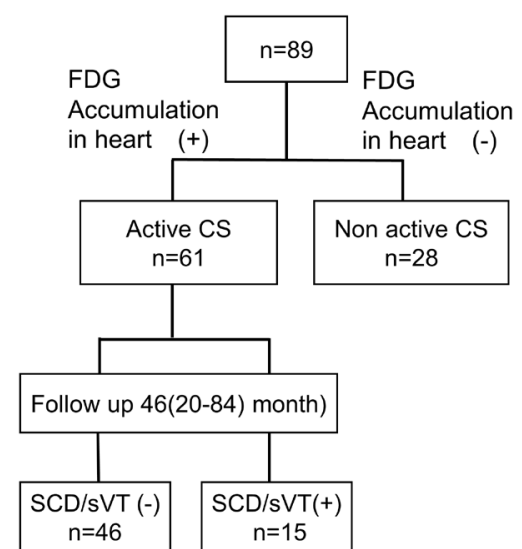

Figure 1 Study design. (A) 8-OHdG as a marker of DNA oxidative damage. Enhanced production of ROS may occur in cardiomyocytes during the active phase of CS, leading to DNA oxidisation (from 2'-deoxyguanosine (blue rod) to 8-OHdG (blue rod with red star)) in the nuclei DNA and mitochondrial DNA, with subsequent excretion of 8-OHdG, in urine via blood. The concentration of 8-0HdG can be measured by ELISA method using an anti-8-OHdG antibody (N45.1). ${ }^{10-12}$ The normal range of U-8-OHdG concentration is defined as $<10 \mathrm{ng} / \mathrm{mg} \cdot \mathrm{Cr}$, as taken from a previous study. ${ }^{9}$ (B) Study protocol. (C) Study flow chart. The 89 patients with CS were divided according to the presence of ${ }^{18} \mathrm{~F}-\mathrm{FDG}$ accumulation in the heart into the following groups: active CS ( $n=61)$ and non-active CS $(n=28)$. Patients with active CS were further divided into the SVT/SCD event $(+)$ group $(n=15)$ and the sVT/SCD event (-) group ( $\mathrm{n}=46) .{ }^{18} \mathrm{~F}-\mathrm{FDG},{ }^{18} \mathrm{~F}$-fluorodeoxyglucose; 8-OHdG, 8-hydroxy-2'-deoxyguanosine; BNP, B-type natriuretic peptide; CRP, C reactive protein; CS, cardiac sarcoidosis; eGFR, estimated glomerular filtration rate; HF, heart failure; IL-6, interleukin 6; LVDd, left ventricular end-diastolic diameter; LVEF, left ventricular ejection fraction; NYHA, New York Heart Association; PET, positron emission tomography; ROC, receiver operating characteristic; ROS, reactive oxygen species; SCD, sudden cardiac death; SUVmax, maximum standardised uptake value; sVT, sustained ventricular tachycardia; TNF- $\alpha$, tumour necrosis factor; TnT, troponin T.

Society's diagnostic and therapeutic guidelines for $\mathrm{CS}^{1014}$ before enrolment. The inclusion criteria were as follows: (1) patients diagnosed with CS, (2) age $\geq 20$ years and (3) New York Heart Association (NYHA) class I and II (on chronic phase). The exclusion criteria were as follows: (1) patients with acute decompensated HF (NYHA class III and IV), (2) acute coronary syndrome, (3) malignancies, (4) infectious diseases, (5) systemic inflammatory diseases and (6) severe renal function disorders (estimated glomerular filtration rate $(\mathrm{eGFR})<30 \mathrm{~mL}$ ).

We investigated whether an oxidative stress marker could predict the prognosis in patients with CS. We previously reported that U-8-OHdG levels significantly increased in patients with NYHA class III and IV chronic HF. ${ }^{9}$ Therefore, patients meeting these criteria were excluded to clarify enhanced inflammatory activity as the cause of increased U-8-OHdG levels in patients with CS.

Written informed consent for participation was obtained from all patients.

Figure 1B depicts the study protocol. Consecutive patients with CS diagnosed according to the Japanese or Heart Rhythm Society guidelines between June 2008 and March 2020 in our hospital were enrolled in this study. On diagnosis or during 
Table 1 Baseline characteristics of all patients with CS

\begin{tabular}{|c|c|c|c|c|}
\hline & Overall $(\mathrm{N}=89)$ & ${ }^{18}$ F-FDG-PET positive $(n=61)$ & ${ }^{18} \mathrm{~F}$-FDG-PET negative $(\mathrm{n}=28)$ & $P$ value \\
\hline Age, years & $65 \pm 11$ & $64 \pm 11$ & $67 \pm 11$ & $0.303 \ddagger$ \\
\hline Male & $38(43)$ & $24(39)$ & $14(50)$ & $0.345^{*}$ \\
\hline \multicolumn{5}{|l|}{ Cardiac symptoms } \\
\hline None & $15(17)$ & $12(20)$ & $3(11)$ & $0.295^{*}$ \\
\hline Syncope & $17(19)$ & $11(18)$ & $6(21)$ & $0705^{*}$ \\
\hline Palpitations & $19(21)$ & $13(21)$ & $6(21)$ & $0.990^{*}$ \\
\hline Dyspnoea & $55(62)$ & $38(62)$ & $17(61)$ & $0.887^{*}$ \\
\hline NYHA class & $1.6 \pm 0.5$ & $1.6 \pm 0.5$ & $1.8 \pm 0.6$ & $0.159 \ddagger$ \\
\hline \multicolumn{5}{|l|}{ ECG/Holter findings } \\
\hline Advanced AVB & $30(34)$ & $18(30)$ & $12(43)$ & $0.219^{*}$ \\
\hline SVT & $22(25)$ & $13(21)$ & $9(32)$ & $0.274^{*}$ \\
\hline NSVT & $17(19)$ & $11(18)$ & $6(21)$ & $0.705^{*}$ \\
\hline CLBBB & $12(13)$ & $7(11)$ & $5(18)$ & $0.670^{*}$ \\
\hline CRBBB & $12(13)$ & $8(13)$ & $4(14)$ & $0.880^{*}$ \\
\hline Q wave & $11(12)$ & $7(11)$ & $4(14)$ & $0.708^{*}$ \\
\hline \multicolumn{5}{|l|}{ Echocardiography } \\
\hline LVDd, mm & $56 \pm 9$ & $55 \pm 8$ & $60 \pm 10$ & $0.005 \ddagger$ \\
\hline LVEF, \% & $40 \pm 14$ & $43 \pm 12$ & $33 \pm 13$ & $0.001 \ddagger$ \\
\hline RWMA score & $2.2(1.5-2.8)$ & $2.0(1.4-2.7)$ & $3.4(2.1-3.6)$ & $0.003 t$ \\
\hline \multicolumn{5}{|l|}{${ }^{18} \mathrm{~F}-\mathrm{FDG}$-PET } \\
\hline Cardiac & $61(69)$ & $61(100)$ & $0(0)$ & $<0.001$ * \\
\hline \multicolumn{5}{|l|}{ Extracardiac } \\
\hline None & $34(38)$ & $24(39)$ & $10(36)$ & $0.743^{*}$ \\
\hline Lung & $8(9)$ & $7(11)$ & $1(4)$ & $0.226^{*}$ \\
\hline Skin & $3(3)$ & $3(5)$ & $0(0)$ & $0.233^{*}$ \\
\hline Liver & $9(10)$ & $8(13)$ & $1(4)$ & $0.166^{*}$ \\
\hline Lymph node & $30(34)$ & $27(44)$ & $3(11)$ & $0.002^{*}$ \\
\hline Other & $4(4)$ & $4(7)$ & $0(0)$ & $0.166^{*}$ \\
\hline Positive LGE on CMR (missing 50/89) & $35(90)$ & $30(90)$ & $5(83)$ & $0.574^{*}$ \\
\hline \multicolumn{5}{|l|}{ Comorbidity } \\
\hline Coronary artery disease & $3(3)$ & $3(5)$ & $0(0)$ & $0.235^{*}$ \\
\hline sVT & $22(25)$ & $13(21)$ & $9(32)$ & $0.271^{*}$ \\
\hline VA & $24(27)$ & $19(31)$ & $5(18)$ & $0.190^{*}$ \\
\hline \multicolumn{5}{|l|}{ Histological diagnosis } \\
\hline Cardiac & $1(1)$ & $1(2)$ & $0(0)$ & $0.496^{*}$ \\
\hline \multicolumn{5}{|l|}{ Extracardiac } \\
\hline Lymph node & $4(4)$ & $2(3)$ & $2(7)$ & $0.414^{*}$ \\
\hline Lung & $10(11)$ & $9(15)$ & $1(4)$ & $0.121^{*}$ \\
\hline Skin & $5(6)$ & $4(7)$ & $1(4)$ & $0.570^{*}$ \\
\hline Eye & $3(3)$ & $3(5)$ & $0(0)$ & $0.233^{*}$ \\
\hline Other & $1(1)$ & $1(2)$ & $0(0)$ & $0.496^{*}$ \\
\hline \multicolumn{5}{|l|}{ Laboratory data } \\
\hline BNP, pg/mL & $89(40-264)$ & $56(30-129)$ & 301 (53-341) & $0.141 \dagger$ \\
\hline $\mathrm{U}-8-\mathrm{OHdG}, \mathrm{ng} / \mathrm{mg} \cdot \mathrm{Cr}$ & $13.8 \pm 6.6$ & $14.9 \pm 7.0$ & $11.0 \pm 4.4$ & $0.015 \ddagger$ \\
\hline \multicolumn{5}{|l|}{ Diagnosis } \\
\hline JCS 2006 & $89(100)$ & $61(100)$ & $28(100)$ & \\
\hline HRS & $20(22)$ & $16(26)$ & $4(14)$ & 0.161 * \\
\hline Isolated CS & $35(39)$ & $24(39)$ & $11(39)$ & $0.996^{*}$ \\
\hline \multicolumn{5}{|l|}{ Treatment } \\
\hline Beta-blocker & $65(73)$ & $45(74)$ & $20(71)$ & $0.817^{*}$ \\
\hline Amiodarone & $24(27)$ & $17(28)$ & $7(25)$ & $0.777^{\star}$ \\
\hline Steroid & $55(62)$ & $47(77)$ & $8(29)$ & $<0.001$ * \\
\hline Device (PM/ICD/CRT) & $57(64)$ & $39(64)$ & $18(64)$ & $0.974^{*}$ \\
\hline
\end{tabular}

The normal range of U-8-OHdG was defined as $<10 \mathrm{ng} / \mathrm{mg} \cdot \mathrm{Cr}$, following a previous study. ${ }^{9}$

Continuous variables are presented as mean \pm SD if normally distributed and median (IQR) if not normally distributed. Categorical variables are presented as number of patients (\%).

${ }^{*} \chi^{2}$ test.

tMann-Whitney U test.

¥t-test.

AVB, complete atrioventricular block; BNP, B-type natriuretic peptide; CLBBB, complete left bundle branch block; CMR, cardiac magnetic resonance; CRBBB, complete right bundle branch block; CRT, cardiac

resynchronisation therapy; CS, cardiac sarcoidosis: ${ }^{18} \mathrm{~F}$-FDG ${ }^{18} \mathrm{~F}$-fluorodeoxyglucose; HRS, Heart Rhythm Society; ICD, implantable cardioverter-defibrillator; JCS, Japanese Circulation Society; LGE, late gadolinium enhancement; LVDd, left ventricular end-diastolic diameter; LVEF, left ventricular ejection fraction; NSVT, non-sustained ventricular tachycardia; NYHA, New York Heart Association; PET, positron emission tomography;

PM, pacemaker; RWMA, regional wall motion abnormality; sVT, sustained ventricular tachycardia; U-8-OHdG, urinary 8-hydroxy-2'-deoxyguanosine; VA, ventricular aneurysm. 


\begin{tabular}{|c|c|c|c|}
\hline & sVT/SCD (-) (n=46) & sVT/SCD $(+)(n=15)$ & $P$ value \\
\hline Age, years & $63 \pm 12$ & $70 \pm 6$ & $0.019^{*}$ \\
\hline Male & $16(35)$ & $8(53)$ & $0.202+$ \\
\hline NYHA class & $1.5 \pm 0.5$ & $1.8 \pm 0.4$ & $0.031^{*}$ \\
\hline LVDd, mm & $54 \pm 7$ & $57 \pm 10$ & $0.150^{*}$ \\
\hline LVEF, \% & $45 \pm 13$ & $40 \pm 9$ & $0.207^{*}$ \\
\hline RWMA score & $2.00(1.34-2.75)$ & $2.59(1.97-2.62)$ & $0.038 \neq$ \\
\hline SBP, mm Hg & $111(104-118)$ & $104(102-113)$ & $0.017 \ddagger$ \\
\hline $\mathrm{HR}, \mathrm{bpm}$ & $71 \pm 10$ & $70 \pm 10$ & $0.776^{*}$ \\
\hline BNP, pg/mL & $50(29-111)$ & 129 (92-265) & $0.001 \ddagger$ \\
\hline $\mathrm{U}-8-\mathrm{OHdG}, \mathrm{ng} / \mathrm{mg} \cdot \mathrm{Cr}$ & $12.7 \pm 6.0$ & $21.0 \pm 6.2$ & $<0.001^{*}$ \\
\hline CRP, mg/dL & $0.09(0.06-0.18)$ & $0.10(0.95-0.12)$ & $0.591 \ddagger$ \\
\hline TNF- $\alpha, p g / m L$ & $1.4(0.9-2.1)$ & $1.6(1.2-1.7)$ & $0.905 \ddagger$ \\
\hline IL-6, pg/mL & $2.3(1.7-3.8)$ & $2.0(1.9-2.3)$ & $0.277 \ddagger$ \\
\hline $\mathrm{ACE}, \mathrm{U} / \mathrm{L}$ & $11.4(8.9-15.5)$ & $9.8(6.3-16.5)$ & $0.289^{*}$ \\
\hline eGFR, $\mathrm{mL} / \mathrm{min} / 1.73 \mathrm{~m}^{2}$ & $65.1 \pm 21.0$ & $61.1 \pm 22.8$ & $0.532^{*}$ \\
\hline $\mathrm{TnT}, \mathrm{ng} / \mathrm{mL}$ & $0.015(0.011-0.019)$ & $0.009(0.007-0.145)$ & $0.977 \ddagger$ \\
\hline SUVmax & $5.9(4.1-7.5)$ & $5.4(4.0-7.0)$ & $0.816^{*}$ \\
\hline $\begin{array}{l}\text { Positive LGE on CMR } \\
\text { (missing 28/61) }\end{array}$ & $24(89)$ & $6(100)$ & $0.392 \dagger$ \\
\hline $\begin{array}{l}\text { Inducibility on VT study } \\
\text { (missing 45/61) }\end{array}$ & $5(56)$ & $3(43)$ & $0.614 \dagger$ \\
\hline \multicolumn{4}{|l|}{ Comorbidity } \\
\hline HT & $19(41)$ & $6(40)$ & $0.929 \dagger$ \\
\hline $\mathrm{DL}$ & $17(37)$ & $6(40)$ & $0.833 \dagger$ \\
\hline DM & $8(17)$ & $5(33)$ & $0.190 \dagger$ \\
\hline Advanced AVB & $15(33)$ & $3(20)$ & $0.352 \dagger$ \\
\hline Coronary artery disease & 2 (4) & $1(7)$ & $0.718+$ \\
\hline VA & $10(22)$ & $9(60)$ & $0.005+$ \\
\hline SVT & $5(11)$ & $8(53)$ & $<0.001 \dagger$ \\
\hline \multicolumn{4}{|l|}{ Treatment } \\
\hline ACEI/ARB & $31(67)$ & $12(80)$ & $0.352 \dagger$ \\
\hline Beta-blocker & $33(72)$ & $12(80)$ & $0.528+$ \\
\hline Amiodarone & $10(22)$ & $7(47)$ & $0.061 \dagger$ \\
\hline Loop diuretics & $12(26)$ & $9(60)$ & $0.016+$ \\
\hline Aldosterone antagonist & $8(17)$ & $5(33)$ & $0.190 \dagger$ \\
\hline Statin & $10(22)$ & $5(33)$ & $0.365+$ \\
\hline Steroid & 34 (74) & $13(87)$ & $0.308+$ \\
\hline Device (PM/ICD/CRT) & $26(57)$ & $13(87)$ & $0.035+$ \\
\hline
\end{tabular}

The normal range of U-8-OHdG was defined as $<10 \mathrm{ng} / \mathrm{mg} \cdot \mathrm{Cr}$, following a previous study. ${ }^{9}$ Continuous variables are presented as mean $\pm S D$ if normally distributed and median (IQR) if not normally distributed. Categorical variables are presented as number of patients (\%).

${ }^{*}$ t-test.

$+\chi^{2}$ test.

¥Mann-Whitney $\mathrm{U}$ test.

ACEl, ACE inhibitor; ARB, angiotensin receptor blocker; AVB, atrioventricular block; BNP, B-type natriuretic peptide; bpm, beats per minute; CMR, cardiac magnetic resonance; CRP, C reactive protein; CRT, cardiac resynchronisation therapy; CS, cardiac sarcoidosis; $\mathrm{DL}$, dyslipidaemia; DM, diabetes mellitus; eGFR, estimated glomerular filtration rate; $\mathrm{HR}$, heart rate; $\mathrm{HT}$, hypertension; ICD, implantable cardioverter-defibrillator; IL-6, interleukin 6; LGE, late gadolinium enhancement; LVDd, left ventricular end-diastolic diameter; LVEF, left ventricular ejection fraction; NYHA, New York Heart Association; PM, pacemaker; RWMA regional wall motion abnormality; SBP, systolic blood pressure; SCD, sudden cardiac death; SUVmax, maximum standardised uptake value; SVT, sustained ventricular tachycardia; TNF- $\alpha$, tumour necrosis factor- $\alpha$; TnT, troponin T; U-8-OHdG, urinary 8-hydroxy-2'-deoxyguanosine;

$\mathrm{VA}$, ventricular aneurysm; $\mathrm{VT}$, ventricular tachycardia.

the chronic phase, the levels of U-8-OHdG, a marker of oxidative DNA damage reflecting CS' inflammatory activity, other biomarkers, and indices of cardiac and renal function were measured.

\section{Standard examinations}

All patients underwent 12-lead ECG, echocardiography, and ${ }^{18}$ F-FDG-PET/CT or ${ }^{67} \mathrm{Ga}$ scintigraphy. All patients were monitored by Holter ECG, an ECG monitor (Nihon Kohden, Tokyo) or an implantable device (implantable cardioverterdefibrillator, cardiac resynchronisation therapy defibrillator or dual-chamber pacemaker). The echocardiographic regional wall motion abnormality (RWMA) score was measured as a marker of myocardial scarring, as previously described. ${ }^{12}$ Ventricular aneurysm (VA) was defined as bulging of the ventricular wall at end-diastole on one of the following imaging tests: transthoracic echocardiography, contrast left ventriculography and MRI. ${ }^{15}$

\section{Evaluation of inflammatory activity due to CS}

All patients underwent ${ }^{18} \mathrm{~F}$-FDG-PET/CT examinations after fasting for 18 hours and the whole body and heart were visualised. Quantitative analysis of ${ }^{18} \mathrm{~F}$-FDG uptake in lesions was performed using maximum standardised uptake value measurements. ${ }^{10-12} 15$

\section{Steroid treatment protocol for patients with active-positive CS}

Corticosteroid treatment (prednisolone) was initiated according to existing Japanese protocols as follows: $30 \mathrm{mg} / \mathrm{day}$ for 4 weeks with gradual tapering of the dose to $5-10 \mathrm{mg}$ every other day over 6 months to establish the minimal effective dose. ${ }^{13}$

\section{Study endpoints}

No patients dropped out during the follow-up period. The appearance of cardiovascular events was confirmed mainly using medical records, autopsy records and death certificates in July 2021. The primary endpoint was defined as the first sVT or SCD. sVT was defined as persistent VT (VT of 100/min or more lasting for $30 \mathrm{~s}$ or more) and electrical storm (appearance of three or more VT episodes within 24 hours). SCD was defined as sudden and unexpected death occurring within 1 hour of symptom onset or occurring in individuals who died within 24 hours of being asymptomatic, presumably due to cardiac arrhythmia or haemodynamic catastrophe. ${ }^{116}$

\section{Measurement of U-8-0HdG and other neurohumoral factors and inflammatory markers}

U-8-OHdG concentrations were measured using an ELISA kit with an anti-8-OHdG antibody, ${ }^{9-12} 15$ and the measured urinary concentrations were corrected based on the creatinine $(\mathrm{Cr})$ values. Serum interleukin 6 (IL-6) concentration was measured using human IL-6 ELISA kit and human IL-6 chemiluminescent enzyme immunoassay. ${ }^{9-12} 15$ B-type natriuretic peptide (BNP) levels were measured using a BNP measurement kit. ${ }^{9-12} 15$

\section{Patient and public involvement}

No participants were involved in setting the research question or the outcome measures. Results from our prospective cohort study are routinely disseminated to the study participants through our research group's websites. We plan to disseminate these findings to the participants and the general public in a press release.

\section{Statistical analysis}

Continuous variables were presented as mean \pm SD when normally distributed and as median and IQR when nonnormally distributed. Between-group differences were compared using unpaired t-test or Mann-Whitney $\mathrm{U}$ test for continuous variables and $\chi^{2}$ test for dichotomous variables, when appropriate. To determine the predictive markers and stratify the risk of sVT/SCD, univariable, 
Table 3 Results of univariable and multivariable analyses for predictors of SVT/SCD in active CS

\begin{tabular}{|c|c|c|c|c|c|c|c|c|c|}
\hline & \multirow{2}{*}{\multicolumn{3}{|c|}{ Univariable }} & \multicolumn{6}{|c|}{ Multivariable } \\
\hline & & & & \multicolumn{3}{|c|}{ Model 1} & \multicolumn{3}{|c|}{ Model 2} \\
\hline & HR & $95 \% \mathrm{Cl}$ & $P$ value & HR & $95 \% \mathrm{Cl}$ & $P$ value & HR & $95 \% \mathrm{Cl}$ & $P$ value \\
\hline Age & 1.064 & 1.006 to 1.125 & 0.030 & 1.071 & 0.977 to 1.174 & 0.145 & & & \\
\hline Male & 2.467 & 0.881 to 6.907 & 0.087 & 4.020 & 0.796 to 20.290 & 0.092 & & & \\
\hline NYHA class & 3.172 & 0.894 to 11.258 & 0.082 & 0.604 & 0.111 to 3.275 & 0.559 & & & \\
\hline LVDd, mm & 1.038 & 0.983 to 1.095 & 0.180 & 0.947 & 0.835 to 1.073 & 0.392 & & & \\
\hline LVEF, \% & 0.977 & 0.937 to 1.019 & 0.281 & & & & & & \\
\hline RWMA score & 1.693 & 0.848 to 3.382 & 0.117 & 0.599 & 0.186 to 1.928 & 0.390 & & & \\
\hline SBP, mm Hg & 0.957 & 0.920 to 0.996 & 0.032 & 0.938 & 0.872 to 1.010 & 0.089 & & & \\
\hline BNP, pg/mL & 1.005 & 1.002 to 1.009 & 0.005 & 1.005 & 0.997 to 1.012 & 0.246 & & & \\
\hline $\mathrm{CRP}, \mathrm{mg} / \mathrm{dL}$ & 0.019 & 0.000 to 19.801 & 0.264 & & & & & & \\
\hline $\mathrm{eGFR}, \mathrm{mL} / \mathrm{min} / 1.73 \mathrm{~m}^{2}$ & 0.994 & 0.970 to 1.018 & 0.611 & & & & & & \\
\hline $\mathrm{U}-8-\mathrm{OHdG}, \mathrm{ng} / \mathrm{mg} \cdot \mathrm{Cr}$ & 1.124 & 1.054 to 1.199 & $<0.001$ & 1.097 & 0.975 to 1.234 & 0.124 & 1.118 & 1.046 to 1.195 & 0.001 \\
\hline Presence of VA & 4.687 & 1.654 to 13.285 & 0.004 & 5.096 & 1.005 to 25.849 & 0.049 & 4.180 & 1.380 to 12.664 & 0.011 \\
\hline History of sVT & 3.940 & 1.426 to 10.883 & 0.008 & 2.323 & 0.493 to 10.942 & 0.287 & 2.728 & 0.904 to 8.234 & 0.075 \\
\hline Inducibility on VT study (missing 45/61) & 0.495 & 0.110 to 2.233 & 0.360 & & & & & & \\
\hline Amiodarone & 2.133 & 0.772 to 5.893 & 0.144 & 0.880 & 0.134 to 5.777 & 0.894 & & & \\
\hline Beta-blocker & 1.426 & 0.402 to 5.059 & 0.583 & & & & & & \\
\hline Loop diuretic & 2.887 & 1.023 to 8.143 & 0.045 & 0.499 & 0.081 to 3.094 & 0.455 & & & \\
\hline Steroid & 2.201 & 0.496 to 9.765 & 0.299 & & & & & & \\
\hline Device (PM/ICD/CRT) & 3.298 & 0.739 to 14.705 & 0.118 & 1.934 & 0.241 to 15.533 & 0.0 .535 & & & \\
\hline
\end{tabular}

BNP, brain natriuretic peptide; CRP, C reactive protein; CRT, cardiac resynchronisation therapy; CS, cardiac sarcoidosis; eGFR, estimated glomerular filtration rate; ICD, implantable cardioverter-defibrillator; LVDd, left ventricular end-diastolic diameter; LVEF, left ventricular ejection fraction; NYHA, New York Heart Association; PM, pacemaker; RWMA, regional wall motion abnormality; SBP, systolic blood pressure; SCD, sudden cardiac death; SVT, sustained ventricular tachycardia; U-8-OHdG, urinary 8-hydroxy-2'-deoxyguanosine; VA, ventricular aneurysm; $\mathrm{VT}$, ventricular tachycardia.

multivariable, receiver operating characteristic (ROC) and Kaplan-Meier analyses were conducted. Univariable analysis was performed using age, sex, NYHA class, left ventricular end-diastolic diameter (LVDd), left ventricular ejection fraction (LVEF), RWMA score, systolic blood pressure (SBP), C reactive protein, eGFR, U-8-OHdG, presence of VA, inducibility on VT study, treatment (amiodarone, beta-blockers, loop diuretics, steroids), history of sVT and device implantation. To predict the primary outcome of sVT/SCD events, multivariable analysis (Cox proportional hazard model) was performed using the covariables with $\mathrm{p}<0.2$ estimated by the univariable analysis (model 1 ). History of sVT is a well-known risk factor for recurrent $\mathrm{sVT}^{17}$; therefore, adjustments were made using backward stepwise approach, including 'history of sVT'. To examine the predictability of U-8-OHdG for sVT/SCD, the ROC method was performed to analyse time-to-event data at $1,2,3,4$ and 5 years and at the final observation point. Each of the maximal cut-off value, sensitivity and specificity was calculated using Youden's index methods. The cut-off value was determined on the ROC curve as the value at which both sensitivity and specificity were well balanced and high. Based on U-8OHdG concentrations, patients were divided into two groups using a maximal cut-off value. Kaplan-Meier analysis was performed to compare survival. Differences in survival curves between the two groups were evaluated using the logrank test. All analyses were performed using SPSS (V.26) and $\mathrm{p}<0.05$ was considered statistically significant.

\section{RESULTS}

\section{Patient background}

Figure 1C shows the flow chart of this study. Eighty-nine consecutive patients diagnosed with CS at Yamaguchi
University Hospital were enrolled on diagnosis and stable period. The 89 enrolled patients with CS were classified into active $(n=61)$ and non-active $(n=28)$ groups based on the abnormal accumulation of ${ }^{18} \mathrm{~F}-\mathrm{FDG}$-PET/CT in the heart (table 1). All 89 (100\%) patients met the 2006 Japanese Circulation Society guidelines, whereas 16 (26\%) patients with active CS and 4 (14\%) patients with non-active CS met the Heart Rhythm Society guidelines, as shown in table 1. Of the 61 patients with active CS, 37 (61\%) were diagnosed with CS with extracardiac sarcoidosis (systemic CS) and $24(39 \%)$ were diagnosed with isolated CS based on ${ }^{18} \mathrm{~F}-\mathrm{F}$ DG-PET/CT (or Ga scintigraphy) and histological examination (cardiac or extracardiac biopsy). There were significant between-group differences in LVDd, LVEF, RWMA score, U-8-OHdG and steroid use. Interestingly, U-8-OHdG levels were significantly lower in the non-active CS group than in the active CS group (active CS $14.9 \pm 7.0 \mathrm{ng} / \mathrm{mg} \cdot \mathrm{Cr}$ vs nonactive CS $11.0 \pm 4.4 \mathrm{ng} / \mathrm{mg} \cdot \mathrm{Cr}, \mathrm{p}=0.015)$. The two groups showed no differences in the other indicators. Thus, these two subgroups largely differed regarding patient characteristics and myocardial inflammatory activity. Therefore, patients with non-active CS were excluded from the present study. The 61 patients with active CS were followed up for a median of 46 months (IQR: 20-84) from the end of registration to the time of data collection regarding sVT/SCD events (July 2021; figure 1B,C).

Table 2 shows the characteristics of patients from the event (sVT/SCD) and non-event groups. Of the 61 patients with active disease, 12 had sVT and 3 showed SCD, yielding a total of 15 patients in the event group, with the remaining 46 in the nonevent group. The two groups showed significant differences in age, NYHA class, RWMA score, SBP, BNP level, U-8-OHdG level, VA, sVT, loop diuretics and device (pacemaker/implantable 
A

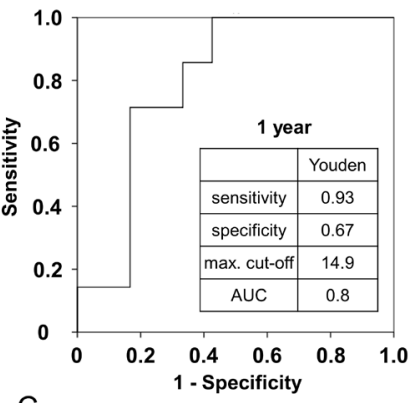

C

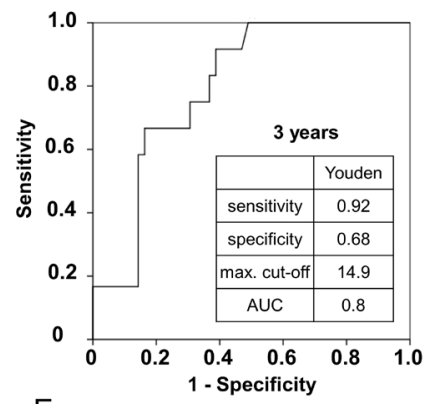

E

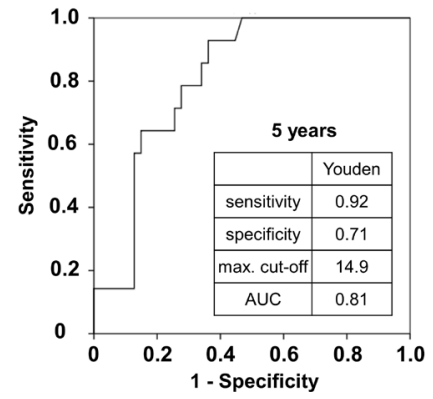

B

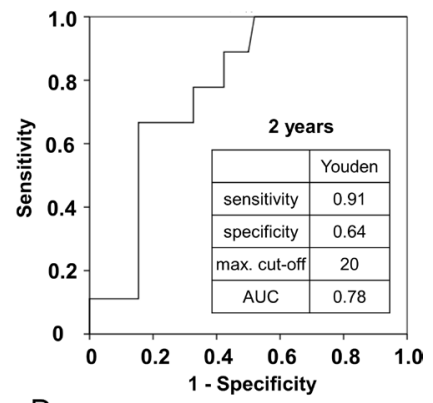

D

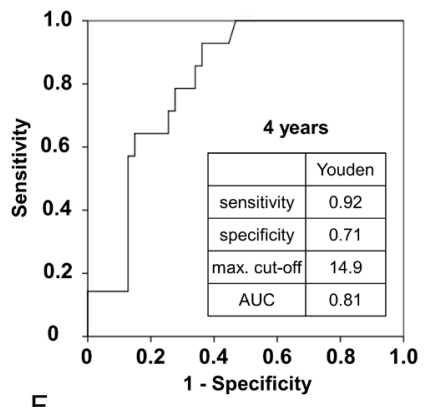

$\mathrm{F}$

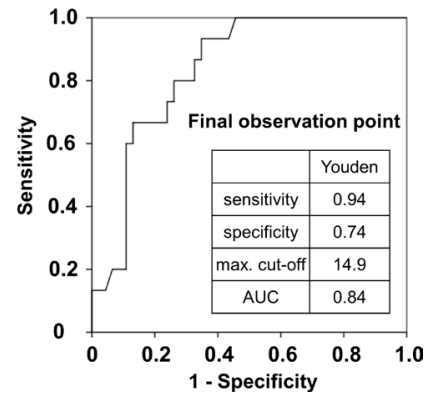

Figure 2 ROC analysis for predicting sustained ventricular tachycardia/sudden cardiac death. ROC method was performed to analyse time-to-event data at 1, 2, 3, 4 and 5 years and at the final observation point. Each of the maximal cut-off value, sensitivity and specificity was calculated by the Youden's index method. The results are shown in each ROC curve. Harrell's c-statistic was calculated as 0.77 . Finally, the cut-off value for U-8-OHdG was decided at $14.9 \mathrm{ng} / \mathrm{mg} \cdot \mathrm{Cr}$ by the Youden's index method. At (A) 1 year, (B) 2 years, (C) 3 years, (D) 4 years, $(E) 5$ years and $(F)$ at the final observation period. AUC, area under the curve; ROC, receiver operating characteristic; U-8-OHdG, urinary 8-hydroxy-2'-deoxyguanosine.

cardioverter-defibrillator/cardiac resynchronisation therapy), but not in the other indicators.

\section{Univariable and multivariable analyses for predictors of first} SVT and SCD complications in active CS

Table 3 shows the results of the univariable and multivariable analyses performed to predict SVT and SCD in the active CS group. Multivariable analysis was performed using the covariables age, gender, NYHA class, LVDd, RWMA score, SBP, BNP, U-8-OHdG, presence of VA, history of VT, amiodarone, loop diuretics and device with $\mathrm{p}<0.2$, as estimated by the univariate analysis (model 1). The backward stepwise approach, including 'history of sVT', showed that U-8-OHdG and presence of VA were stronger predictors than history of sVT (model 2). Finally, U-8-OHdG was associated with a 1.118-fold increased risk per ng/mg.Cr of sVT/SCD (HR, 1.118; 95\% CI 1.046 to 1.195 ; $\mathrm{p}=0.001$ ), and presence of VA was associated with a 4.18 -fold

A. SVT/SCD (including patients with VT history) B. SVT (including patients with VT history)

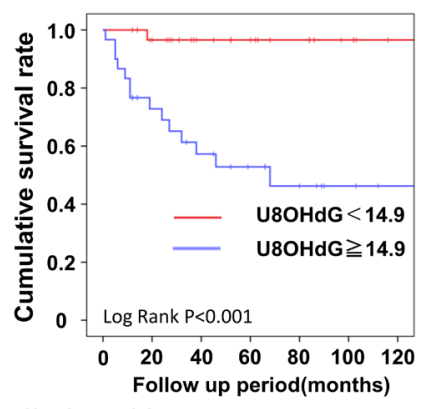

Number at risk
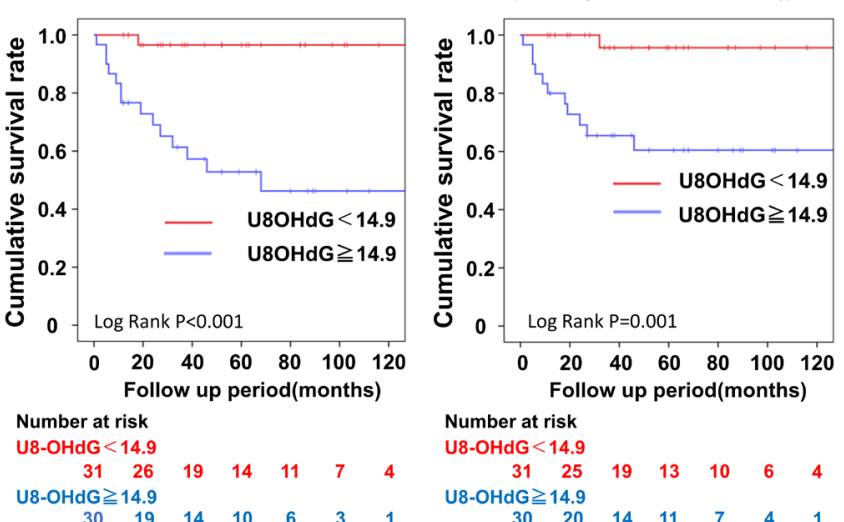

Number at risk

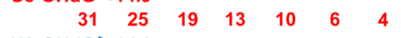

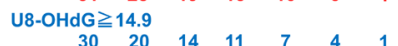

C. SCD (including patients with VT history)

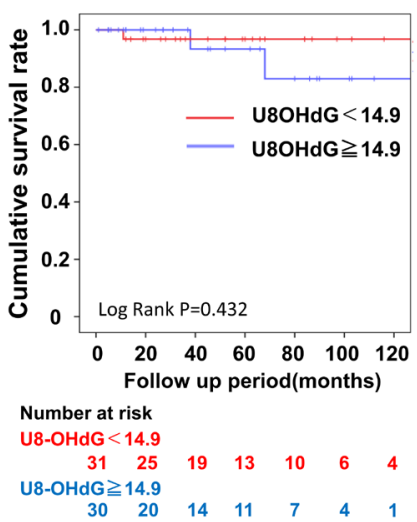

D. SVT/SCD (excluding patients with VT history)

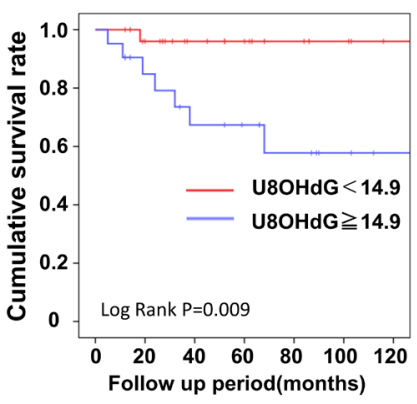

Number at risk

U8-OHdG $<14.9$

$\begin{array}{lllllll}27 & 22 & 16 & 11 & 8 & 6 & 3\end{array}$

$30 \quad 20$

Figure 3 Survival analyses for patients with active CS divided into two groups based on the cut-off value for U-8-OHdG. The primary outcome was a composite of SVT/SCD in our cohort. Cumulative eventfree survival rates of a composite (A), SVT (B), SCD (C) and a composite of SVT/SCD excluding patients with a history of VT (D) were estimated using the Kaplan-Meier method. CS, cardiac sarcoidosis; SCD, sudden cardiac death; sVT, sustained ventricular tachycardia; $\mathrm{U}-8-\mathrm{OHdG}$, urinary 8-hydroxy-2'-deoxyguanosine; $\mathrm{VT}$, ventricular tachycardia.

increased risk of sVT/SCD, and VA was an independent predictor of sVT/SCD (HR, 4.180; 95\% CI 1.380 to $12.664 ; \mathrm{p}=0.011$ ).

Time-dependent ROC analysis for the optimal cut-off value of U-8-OHdG as a predictor of SVT/SCD in active CS

Figure 2A-F shows the ROC curves at each point. Each of the optimal cut-off value, sensitivity and specificity was calculated using Youden's index methods and the results are shown in each ROC curve. Harrell's c-index was calculated as 0.77 and the area under the curve was $\sim 0.8$ at each point. The results suggest that U-8-OHdG has high predictability for sVT/SCD. Finally, the cutoff value for U-8-OHdG was decided at $14.9 \mathrm{ng} / \mathrm{mg} \cdot \mathrm{Cr}$ using the Youden's index method.

\section{Kaplan-Meier analysis using the cut-off value of U-8-OHdG}

When the patients were grouped based on a cut-off U-8-OHdG value of $14.9 \mathrm{ng} / \mathrm{mg} \cdot \mathrm{Cr}$, Kaplan-Meier analysis showed that the group with levels $\geq 14.9 \mathrm{ng} / \mathrm{mg}$. Cr (higher U-8-OHdG) had a significantly poorer prognosis than the group with levels lower than $14.9 \mathrm{ng} / \mathrm{mg} \cdot \mathrm{Cr}$ (lower U-8-OHdG; figure 3A-C). History of sVT is a well-known risk of recurrent sVT/SCD. ${ }^{17}$ In a sensitivity analysis excluding patients with a history of sVT, Kaplan-Meier analysis showed that the group with levels $\geq 14.9 \mathrm{ng} / \mathrm{mg}$. $\mathrm{Cr}$ or 
A. SVT/SCD (including patients with VT history)

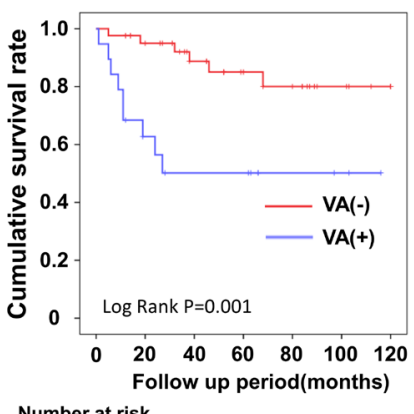

Number at risk

VA(-) $\quad \begin{array}{lllllll}42 & 35 & 26 & 17 & 14 & 8 & 5\end{array}$

$\begin{array}{llllllll}\mathrm{VA}+ & 19 & 10 & 7 & 7 & 3 & 2 & 0\end{array}$

C. SCD (including patients with VT history)

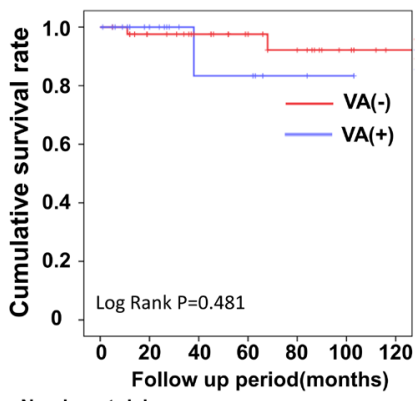

Number at risk

$\begin{array}{llllllll}\text { VA(-) } & 42 & 34 & 28 & 19 & 15 & 9 & 5\end{array}$

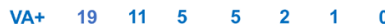

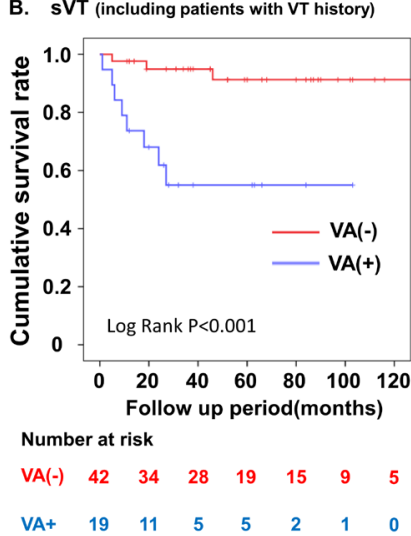

D. SVT/SCD (excluding patients with VT history)

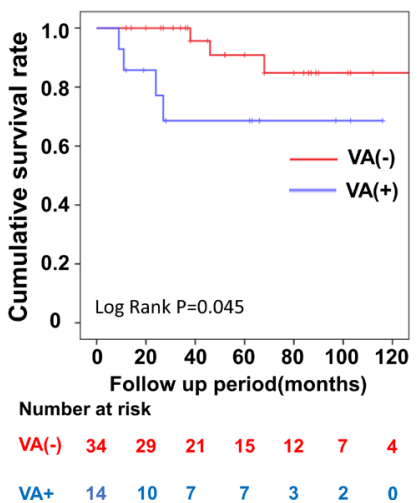

Figure 4 Survival analyses for patients with CS divided into two groups based on the presence of VA. The primary outcome was a composite of sVT/SCD in our cohort. The cumulative event-free survival rates of a composite (A), sVT (B), SCD (C) and a composite of sVT/SCD excluding patients with a history of VT (D) were estimated using the Kaplan-Meier method. CS, cardiac sarcoidosis; SCD, sudden cardiac death; sVT, sustained ventricular tachycardia; VA, ventricular aneurysm; VT, ventricular tachycardia.

more had a significantly poorer prognosis than the group with levels <14.9 ng/mg. Cr (log-rank test, $\mathrm{p}=0.009$; figure 3D). The incidence rates of sVT/SCD (events per 100 patient-year) were 0.6 (95\% CI 0.015 to 3.303 ) and 12.3 (95\% CI 6.739 to 20.681) for lower and higher U-8-OHdG, respectively (figure $3 \mathrm{~A}$ ).

\section{Kaplan-Meier analysis using the presence of VA}

Kaplan-Meier analysis showed that the CS group with VA had significantly poorer prognoses than the CS group without VA (figure 4A-C). In a sensitivity analysis excluding patients with a history of sVT, Kaplan-Meier analysis showed that the VA group had significantly poorer prognoses than the group without VA (log-rank test, $\mathrm{p}=0.045$; figure 4D). The incidence rates of $\mathrm{sVT} /$ SCD (events per 100 patient-year) were 2.7 (95\% CI 1.000 to 5.932) and 14.5 (95\% CI 6.629 to 27.519$)$ for those without and with VA, respectively (figure 4A).

\section{Kaplan-Meier analysis using U-8-OHdG and presence of VA}

When the patients were divided into three groups based on the cut-off value of U-8-OHdG and the presence of VA, patients showing U-8-OHdG concentrations $\geq 14.9 \mathrm{ng} / \mathrm{mg} \cdot \mathrm{Cr}$ and VA had a significantly higher sVT/SCD risk (log-rank, $\mathrm{p}<0.001$ vs patients with neither; events per 100 patient-year, 31.0; figure 5).
A. SVT/SCD (including patients with VT history)

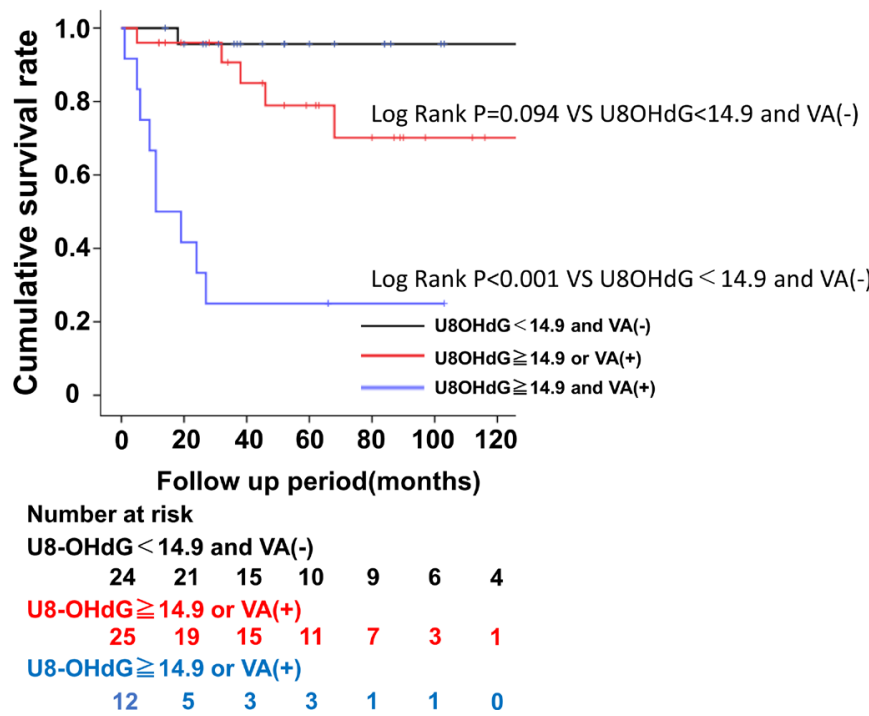

Figure 5 Risk stratification based on U-8-OHdG and VA. Patients showing U-8-OHdG concentration $\geq 14.9 \mathrm{ng} / \mathrm{mg} \cdot \mathrm{Cr}$ and presence of VA had a significantly highest risk of sVT/SCD (log-rank, $p<0.001$ vs patients with neither, events per 100 patient-year, 31.0). SCD, sudden cardiac death; sVT, sustained ventricular tachycardia; U-8-OHdG, urinary 8-hydroxy-2'-deoxyguanosine; VA, ventricular aneurysm; VT, ventricular tachycardia.

\section{DISCUSSION}

\section{Overview}

The most important finding of this prospective cohort study is that U-8-OHdG measurements and presence of VA are useful in predicting sVT/SCD in patients with active CS. This is supported by the following findings: (1) During the observation period, the group with sVT/SCD events had a higher mean U-8-OHdG concentration and a higher VA complication rate than those without sVT/SCD events. (2) Univariable analysis showed that age, SBP, BNP, U-8-OHdG, presence of VA, history of sVT and loop diuretics were significantly correlated with sVT/SCD. However, the multivariable analysis revealed that U-8-OHdG level and presence of VA were independent prognostic factors for sVT/SCD. (3) ROC analysis showed that the cut-off value of U-8-OHdG for predicting first sVT/SCD was $14.9 \mathrm{ng} / \mathrm{mg} \cdot \mathrm{Cr}$. (4) Patients with U-8-OHdG concentration $\geq 14.9 \mathrm{ng} / \mathrm{mg} \cdot \mathrm{Cr}$ and presence of VA had a significantly higher risk of sVT/SCD. This is the first report identifying a biomarker of oxidative stress that is clinically useful for prediction of sVT/SCD in patients with CS.

\section{U-8-OHdG and presence of VA provide relevant information about sVT's arrhythmia substrates}

Ventricular arrhythmias and SCD are believed to be driven by two separate but related mechanisms; one such mechanism is active inflammation, but in the absence of this, scar burden can cause arrhythmias. ${ }^{18}$ In this study, U-8-OHdG levels were significantly higher in patients with active CS than in patients with non-active CS (table 1), which strongly suggests that 8-OHdG production was enhanced by CS-related inflammation.

We previously reported that (1) U-8-OHdG levels are significantly correlated with the inflammatory activity observed on ${ }^{18}$ F-FDG-PET in patients with $\mathrm{CS}^{10}$; and (2) U-8-OHdG levels remarkably increased in patients with a history of sVT and were positively correlated with the degree of oxidative stress and 
myocardial fibrosis area in myocardial biopsy tissue. ${ }^{12}$ These findings suggest that myocardial oxidative stress and inflammation are closely related to the onset of ventricular arrhythmia due to triggered activity. ${ }^{19-24}$ In addition, a high U-8-OHdG level also indicates a high degree of myocardial fibrosis, which is considered to contribute to the formation and maintenance of the re-entry circuit. ${ }^{825} 26$

On the other hand, the presence of VA may indicate the appearance of relatively large scars to varying degrees. ${ }^{15}$ In this study, the RWMA score used as an index of scar tissue by echocardiography was significantly higher in the sVT/SCD event group than in the sVT/SCD non-event group (table 2). The presence of VA is prone to cause scar-related VT due to the presence of ventricular scar and myocardial fibrosis capable of sustaining many re-entrant circuits. ${ }^{85-27}$

In a sensitivity analysis excluding patients with a history of sVT, Kaplan-Meier analysis showed that the group with levels $\geq 14.9 \mathrm{ng} / \mathrm{mg} \cdot \mathrm{Cr}$ of U-8OHdG had significantly poorer prognoses than the group with levels $<14.9 \mathrm{ng} / \mathrm{mg} \cdot \mathrm{Cr}$ (figure 3D) and that the CS group with VA had significantly poorer prognoses than the CS group without VA (figure 4D). These results strongly suggested that U-8-OHdG and presence of VA are independent predictors of sVT/SCD.

\section{U-8-OHdG as a marker of myocardial oxidative stress}

To investigate whether the whole-body burden of sarcoidosis influences the levels of U-8-OHdG in patients with active CS $(n=61$; table 1$)$, we compared U-8-OHdG levels between systemic $(n=37)$ and isolated $(n=24)$ CS and found no difference in U-8-OHdG levels between the two types (online supplemental S-Figure 1). One possible reason for the heart's increased 8-OHdG production may be that it contains more mitochondria than extracardiac organs, and therefore pathophysiological conditions' effect on mitochondrial reactive oxygen species generation is larger in heart tissue. ${ }^{12}$ We previously reported that U-8-OHdG levels were significantly lower in patients with active pulmonary sarcoidosis than in patients with active CS. ${ }^{12}$ These findings support that U-8-OHdG is not susceptible to the systemic burden of extracardiac sarcoidosis.

\section{Other predictors of $S V T / S C D$}

It has been reported that positive late gadolinium enhancement (LGE) on cardiac magnetic resonance (CMR) and the inducibility of VT on a VT study were associated with lethal arrhythmia. ${ }^{4-8}$ However, in our cohort, positive LGE and VT inducibility were not associated with future sVT/SCD in the univariable analysis (table 3). However, too much data regarding CMR and the VT study were missing to conclude whether LGE and VT inducibility can predict sVT/SCD in our cohort.

A history of sVT is a well-known risk factor for recurrent sVT. ${ }^{17}$ The univariable analysis showed that history of sVT was significantly associated with future $\mathrm{sVT} / \mathrm{SCD}(\mathrm{p}=0.008)$ in our cohort study, but the multivariable analysis did not $(\mathrm{p}=0.075)$, which represents a lack of statistical power. It is noteworthy that U-8-OHdG and presence of VA were stronger predictors than history of sVT in this cohort study.

\section{Limitations}

This study had several limitations. First, this was a single-centre, prospective cohort study with few cases. Further large-scale studies are needed to establish the usefulness of U-8-OHdG and the presence of VA as prognostic factors for SVT/SCD in CS. Second, CMR and VT study were missing for most patients with
CS. Therefore, LGE and VT inducibility were not included in the multivariable analysis.

\section{CONCLUSIONS}

U-8-OHdG and presence of VA were powerful predictors of first sVT/SCD in CS and were useful for stratifying cardiac events and providing relevant information about VT's substrates. These results will also provide a foundation for more detailed studies on early prognostic markers for sVT/SCD in CS.

\section{Key messages}

What is already known on this subject?

- Patients with cardiac sarcoidosis (CS) are at an increased risk of sudden cardiac death, which accounts for most lethal ventricular arrhythmias.

- The inflammation caused by CS strongly enhances cardiac oxidative stress in cardiac tissue.

- The level of urinary 8-hydroxy-2'-deoxyguanosine (U-8$\mathrm{OHdG}$ ), an oxidative stress marker, reflects the inflammatory activity of CS.

\section{What might this study add?}

- U-8-OHdG measurements and presence of ventricular aneurysm (VA) are powerful predictors of the first sustained ventricular tachycardia/sudden cardiac death in patients with CS.

- U-8-OHdG measurements and presence of VA are useful in the stratification of cardiac events.

\section{How might this impact on clinical practice?}

- U-8-OHdG provides relevant information that oxidative stress and acute inflammation can induce lethal arrhythmia in patients with CS.

Contributors Acting as guarantor: SK. Concept and design: SK. Acquisition, analysis or interpretation of data: RY, SK, YYa, YN, SF, TN, HI, MF, YYo, TO, KS. Drafting of the manuscript: RY and SK. Statistical analysis: RY, RK and SK. Obtained funding: SK. Supervision: MY.

Funding This work was funded by Grants-in-Aid for Scientific Research from the Ministry of Education in Japan (grant number 23592256 to SK) and SENSHIN Medical Research Foundation Grant 2020 (to SK).

Competing interests None declared.

Patient consent for publication Consent obtained directly from patient(s).

Ethics approval This study involves human participants and was approved by the institutional review committee of Yamaguchi University Hospital (\#H19-87) on 16 April 2008. Participants gave informed consent to participate in the study before taking part.

Provenance and peer review Not commissioned; externally peer reviewed. Data availability statement Data are available upon reasonable request.

Supplemental material This content has been supplied by the author(s). It has not been vetted by BMJ Publishing Group Limited (BMJ) and may not have been peer-reviewed. Any opinions or recommendations discussed are solely those of the author(s) and are not endorsed by BMJ. BMJ disclaims all liability and responsibility arising from any reliance placed on the content. Where the content includes any translated material, BMJ does not warrant the accuracy and reliability of the translations (including but not limited to local regulations, clinical guidelines, terminology, drug names and drug dosages), and is not responsible for any error and/or omissions arising from translation and adaptation or otherwise.

Open access This is an open access article distributed in accordance with the Creative Commons Attribution Non Commercial (CC BY-NC 4.0) license, which permits others to distribute, remix, adapt, build upon this work non-commercially, and license their derivative works on different terms, provided the original work is 
properly cited, appropriate credit is given, any changes made indicated, and the use is non-commercial. See: http://creativecommons.org/licenses/by-nc/4.0/.

\section{ORCID iD}

Shigeki Kobayashi http://orcid.org/0000-0001-5407-2999

\section{REFERENCES}

1 Ekström K, Lehtonen J, Nordenswan H-K, et al. Sudden death in cardiac sarcoidosis: an analysis of nationwide clinical and cause-of-death registries. Eur Heart J 2019;40:3121-8.

2 Kobayashi Y, Nagai T, Takenaka S, et al. Long-Term prognostic significance of ventricular repolarization dispersion in patients with cardiac sarcoidosis. Am J Cardiol 2021;152:125-31.

3 Narasimhan B, Patel N, Ho K, et al. Incidence and predictors of sudden cardiac arrest in sarcoidosis: a nationwide analysis. JACC Clin Electrophysiol 2021;7:1087-95.

4 Franke KB, Marshall H, Kennewell P, et al. Risk and predictors of sudden death in cardiac sarcoidosis: a systematic review and meta-analysis. Int J Cardiol 2021;328:130-40

5 Zipse MM, Tzou WS, Schuller JL, et al. Electrophysiologic testing for diagnostic evaluation and risk stratification in patients with suspected cardiac sarcoidosis with preserved left and right ventricular systolic function. J Cardiovasc Electrophysiol 2019;30:1939-48.

6 Blankstein R, Osborne M, Naya M, et al. Cardiac positron emission tomography enhances prognostic assessments of patients with suspected cardiac sarcoidosis. J Am Coll Cardiol 2014;63:329-36.

7 Patel MR, Cawley PJ, Heitner JF, et al. Detection of myocardial damage in patients with sarcoidosis. Circulation 2009;120:1969-77.

8 Muser D, Santangeli P, Liang JJ, et al. Characterization of the electroanatomic substrate in cardiac sarcoidosis: correlation with imaging findings of scar and inflammation. JACC Clin Electrophysiol 2018;4:291-303.

9 Kobayashi S, Susa T, Tanaka T, et al. Urinary 8-hydroxy-2'-deoxyguanosine reflects symptomatic status and severity of systolic dysfunction in patients with chronic heart failure. Eur J Heart Fail 2011;13:29-36.

10 Kobayashi S, Myoren T, Oda S, et al. Urinary 8-hydroxy-2'-deoxyguanosine as a novel biomarker of inflammatory activity in patients with cardiac sarcoidosis. Int J Cardiol 2015:190:319-28.

11 Myoren T, Kobayashi S, Oda S, et al. An oxidative stress biomarker, urinary 8-hydroxy2'-deoxyguanosine, predicts cardiovascular-related death after steroid therapy for patients with active cardiac sarcoidosis. Int J Cardiol 2016;212:206-13.

12 Ishiguchi H, Kobayashi S, Myoren T, et al. Urinary 8-hydroxy-2'-deoxyguanosine as a myocardial oxidative stress marker is associated with ventricular tachycardia in patients with active cardiac sarcoidosis. Circulation 2017;10:e006764.
13 Terasaki F, Azuma A, Anzai T, et al. JCS 2016 Guideline on Diagnosis and Treatment of Cardiac Sarcoidosis — Digest Version —. Circ J 2019;83:2329-88.

14 Birnie DH, Sauer WH, Bogun F, et al. Hrs expert consensus statement on the diagnosis and management of arrhythmias associated with cardiac sarcoidosis. Heart Rhythm 2014;11); :1304-23. 1.

15 Nanno T, Kobayashi S, Yoshitomi R, et al. Detection of active inflammation status around ventricular aneurysms in patients with cardiac sarcoidosis. Circ $J$ 2019;83:2494-504.

16 Yousuf 0 , Chrispin J, Tomaselli GF, et al. Clinical management and prevention of sudden cardiac death. Circ Res 2015;116:2020-40.

17 Priori SG, Blomström-Lundqvist C, Mazzanti A, et al. 2015 ESC guidelines for the management of patients with ventricular arrhythmias and the prevention of sudden cardiac death. Eur Heart J 2015;36:2793-867.

18 Viwe M, Nery P, Birnie DH. Management of ventricular tachycardia in patients with cardiac sarcoidosis. Heart Rhythm 02 2021;2:412-22.

19 Murakami W, Kobayashi S, Susa T, et al. Recombinant atrial natriuretic peptide prevents aberrant $\mathrm{Ca} 2+$ leakage through the ryanodine receptor by suppressing mitochondrial reactive oxygen species production induced by isoproterenol in failing cardiomyocytes. PLoS One 2016;11:e0163250.

20 Bovo E, Lipsius SL, Zima AV. Reactive oxygen species contribute to the development of arrhythmogenic $\mathrm{Ca}^{2+}$ waves during $\beta$-adrenergic receptor stimulation in rabbit cardiomyocytes. J Physio/ 2012;590:3291-304.

21 Willis BC, Salazar-Cantú A, Silva-Platas C, et al. Impaired oxidative metabolism and calcium mishandling underlie cardiac dysfunction in a rat model of postacute isoproterenol-induced cardiomyopathy. Am J Physiol Heart Circ Physiol 2015;308:H467-77.

22 Yano M, Okuda S, Oda T, et al. Correction of defective interdomain interaction within ryanodine receptor by antioxidant is a new therapeutic strategy against heart failure. Circulation 2005; 112:3633-43.

23 Anderson ME, Hodgson-Zingman DM. Ventricular tachycardia in patients with heart failure. In: Zipes DP, Jalife J, eds. Cardiac electrophysiology: from cell to bedside. fifth edition. WB Sauders, 2009: 707-16.

24 Curran J, Brown KH, Santiago DJ, et al. Spontaneous Ca waves in ventricular myocytes from failing hearts depend on Ca2+-calmodulin-dependent protein kinase II. J Mol Cell Cardiol 2010;49:25-32.

25 Kumar S, Barbhaiya C, Nagashima K. Characterization of ventricular substrate and outcomes of catheter ablation. Circ Arrhythm Electrophysiol 2015;8:87-93.

26 Lull RJ, Dunn BE, Gregoratos G, et al. Ventricular aneurysm due to cardiac sarcoidosis with surgical cure of refractory ventricular tachycardia. Am J Cardiol 1972;30:282-7.

27 Miyazawa K, Yoshikawa T, Takamisawa I, et al. Presence of ventricular aneurysm predicts poor clinical outcomes in patients with cardiac sarcoidosis. Int J Cardiol 2014;177:720-2. 